Letters

\title{
DENTAL NURSES ARE PAID NO MORE THAN SHOP ASSISTANTS
}

\section{Dear Editor,}

I am writing in response to a recent dental survey which I came across (Fig. 1). As a dental nurse in the North West I was disheartened to see the average wage of a registered dental nurse's salary is $£ 15,000$ per year.

Since registration began in 2009 it still seems that the dental nurse has not got the 'professional' recognition they deserve. With increasing workload and the prospect of getting sued it makes the dental nurse role seem undesirable. Why would someone want to work for this amount when they have less stress and no professional outgoings as someone who works in retail on the same pay?

I know that a few people will see this letter as negative but at the end of the day the professional status affects the way we behave
24 hours a day and let's face it we all have bills

to pay. Don't get me wrong I love my job and in qualification to be on nearly the same wage as someone who possesses no qualifications.

With a lot of dental nurses who are low paid it must be a real financial struggle, with the extra bills like CPD, indemnity and

\section{'IT SEEMS UNFAIR THAT THE DENTAL NURSE}

PASSES A RECOGNISED QUALIFICATION TO

BE ON NEARLY THE SAME WAGE AS SOMEONE

\section{WHO POSSESSES NO QUALIFICATIONS.'}

the role that I am in I now feel very, very lucky that I am paid a decent hourly rate, I just feel really disheartened for the dental nurses that are paid near the national minimum wage. It seems unfair that the dental nurse passes a recognised registration - it all seems unfair. I would like to see a wage pay scale that reflects a dental nurse's skills and knowledge.

Claire Strirrup RDN

\section{DRN}

\begin{tabular}{|c|c|c|c|c|c|c|c|}
\hline & & Dental Nurse & Dental Receptionist & Treatment Co-ordinator & Practice Manager & Hygienist \& Therapist & Associate Dentist \\
\hline \multirow[b]{2}{*}{ East Midlands } & Quartile Range & $f 14 k-f 29 k$ & f15k-f27k & $£ 22 k-f 29 k$ & f19k-f28k & £30k-f49k & f30k-f74k \\
\hline & Average & $f 18.7 \mathrm{k}$ & $\mathrm{f} 18 \mathrm{k}$ & $f 24 \mathrm{k}$ & $\mathrm{f24.5 \textrm {k }}$ & $£ 40 \mathrm{k}$ & f54k \\
\hline \multirow[b]{2}{*}{ East of England } & Quartile Range & $f 15 k-f 29 k$ & f14k-f25k & f19k-f28k & $f 25 k-f 26 k$ & $f 30 k-f 49 k$ & f50k-f80k \\
\hline & Average & f19k & $£ 17 \mathrm{k}$ & $f 23.5 \mathrm{k}$ & $\mathrm{f25.5 \textrm {k }}$ & f39k & $\mathrm{f60k}$ \\
\hline \multirow[b]{2}{*}{ London } & Quartile Range & f16k-f31k & $f 18 k-f 29 k$ & $\mathrm{f} 25 \mathrm{k}-\mathrm{f} 31 \mathrm{k}$ & $f 22 k-f 40 k$ & f53k-f60k & f50k-f90k \\
\hline & Average & $\mathrm{f21 \textrm {k }}$ & $\mathrm{f20k}$ & $£ 28 \mathrm{k}$ & $£ 30 \mathrm{k}$ & $\mathrm{f51.5 \textrm {k }}$ & $\mathrm{f} 80 \mathrm{k}$ \\
\hline \multirow[b]{2}{*}{ North East } & Quartile Range & f13k-f24k & f13k-f23k & f16k-f25k & f21k-f26k & $£ 29 k-f 50 k$ & $£ 30 k-£ 72 k$ \\
\hline & Average & f15k & f15k & $f 22 \mathrm{k}$ & $\mathrm{f21.5 \textrm {k }}$ & $£ 42 \mathrm{k}$ & $£ 43 \mathrm{k}$ \\
\hline \multirow[b]{2}{*}{ N. Ireland } & Quartile Range & f14k-f22k & f14k-f24k & f17k-f26k & f18k-f29k & f35k-f53k & $£ 34 k-f 76 k$ \\
\hline & Average & $£ 14.5 \mathrm{k}$ & $\mathrm{f} 19 \mathrm{k}$ & $f 23 \mathrm{k}$ & $\mathrm{f24k}$ & $£ 45 \mathrm{k}$ & $£ 45 \mathrm{k}$ \\
\hline \multirow[b]{2}{*}{ North West } & Quartile Range & f14k-f22k & f13k-f23k & f16k-f24k & f15k-f25k & $f 30 k-f 42 k$ & $f 30 k-f 72 k$ \\
\hline & Average & f15k & $\mathrm{f17 \textrm {k }}$ & $\mathrm{f} 18.5 \mathrm{k}$ & $£ 24 \mathrm{k}$ & $£ 40 \mathrm{k}$ & $£ 42 \mathrm{k}$ \\
\hline \multirow[b]{2}{*}{ Scotland } & Quartile Range & $\mathrm{f} 13-\mathrm{f} 23 \mathrm{k}$ & f13-f24k & f15k-f26k & f23k-f30k & $f 23 k-f 46 k$ & $f 32 k-f 75 k$ \\
\hline & Average & $£ 18 \mathrm{k}$ & $£ 19.5 \mathrm{k}$ & $\mathrm{f25k}$ & $\mathrm{f25 \textrm {k }}$ & $\mathrm{f35k}$ & $£ 43 \mathrm{k}$ \\
\hline \multirow[b]{2}{*}{ South East } & Quartile Range & f13k-f27k & f13k-f28k & f19k-f28k & f23k-f31k & f32k-f55k & $f 34 k-f 72 k$ \\
\hline & Average & $\mathrm{f} 18 \mathrm{k}$ & $\mathrm{f} 18 \mathrm{k}$ & $f 24 \mathrm{k}$ & $\mathrm{f28 \textrm {k }}$ & $£ 42 \mathrm{k}$ & $£ 45 \mathrm{k}$ \\
\hline \multirow[b]{2}{*}{ South West } & Quartile Range & f13k-f23k & f13k-f26k & f18k-f28k & f17k-f30k & f35k-f50k & $f 34 k-f 80 k$ \\
\hline & Average & $\mathrm{f} 18 \mathrm{k}$ & $£ 17.5 \mathrm{k}$ & $\mathrm{f24k}$ & $£ 26 \mathrm{k}$ & $£ 40 \mathrm{k}$ & $£ 45 \mathrm{k}$ \\
\hline \multirow[b]{2}{*}{ Wales } & Quartile Range & f12k-f20k & f13k-f24k & f15k-f24k & $\mathrm{f} 17 \mathrm{k}-\mathrm{f} 30 \mathrm{k}$ & $f 30 k-f 42 k$ & $f 30 k-f 69 k$ \\
\hline & Average & $\mathrm{f} 16 \mathrm{k}$ & $£ 17.5 \mathrm{k}$ & $\mathrm{f} 20 \mathrm{k}$ & $\mathrm{f22k}$ & $£ 39 \mathrm{k}$ & $£ 44 \mathrm{k}$ \\
\hline \multirow[b]{2}{*}{ West Midlands } & Quartile Range & f13k-f24k & $f 13 k-f 26 k$ & $£ 16.5 k-f 27 k$ & f18k-f28k & $£ 28 k-f 51 k$ & f35k-f80k \\
\hline & Average & $f 16.5 \mathrm{k}$ & f17k & f23k & $£ 24.5 \mathrm{k}$ & $£ 38 \mathrm{k}$ & $£ 45 \mathrm{k}$ \\
\hline \multirow[b]{2}{*}{ Yorkshire and the Humber } & Quartile Range & f15k-f23k & $f 13 k-f 25.5 k$ & f14k-f28k & $\mathrm{f} 19 \mathrm{k}-\mathrm{f} 32 \mathrm{k}$ & $f 25 k-£ 40 k$ & $f 32 k-f 78 k$ \\
\hline & Average & f17k & $£ 18.2 \mathrm{k}$ & $£ 21 \mathrm{k}$ & $\mathrm{f} 24.5 \mathrm{k}$ & f35k & $£ 45 \mathrm{k}$ \\
\hline
\end{tabular}

